\title{
Divide and conquer: identifying acute respiratory distress syndrome subphenotypes
}

\author{
Manu Shankar-Hari, ${ }^{1,2}$ Daniel F McAuley ${ }^{3,4}$
}

The acute respiratory distress syndrome (ARDS) definition identifies patients with acute onset hypoxaemia and respiratory failure, who have bilateral opacities on chest radiograph that are not fully explained by cardiac failure or fluid overload. ${ }^{1}$ ARDS is a common illness that accounts for approximately $10 \%$ of critical care admissions and $20 \%$ of patients requiring mechanical ventilation. ${ }^{2}$ The hospital mortality in patients with ARDS remains high, increasing from approximately $35 \%$ for those with mild disease to $46 \%$ for those with severe ARDS. ${ }^{2}$ This high mortality has remained relatively unchanged in the last 20 years. ${ }^{3}$ To date, despite decades of research, there is no pharmacological treatment that can modify the underlying biological mechanisms implicated in ARDS and improve patient outcomes. ${ }^{4}$ Within ARDS populations, there is substantial biological and outcome heterogeneity, with observed differences in dominant pathogenic mechanisms, treatment responses and outcomes. ${ }^{5-7}$ Identifying ARDS subphenotypes based on pathogenic mechanisms that determine treatment responses irrespective of ARDS severity is defined as predictive enrichment. ${ }^{78}$ The identification of such ARDS subphenotypes will enable improved trial design in ARDS by selecting patients based on responder characteristics to therapeutic interventions, hopefully resulting in improved outcomes. $^{6}$

In Thorax, Bos et al report a cohort study in 700 ARDS patients, testing the hypothesis that ARDS subgroups exist due to differences in biological characteristics. ${ }^{9}$ In this retrospective analysis of a prospectively collected cohort, 20 biomarkers

\footnotetext{
${ }^{1}$ Department of Critical Care Medicine, Guy's and St Thomas' NHS Foundation Trust, London, UK

${ }^{2}$ Division of Infection and Immunity, King's College London, London, London, UK

${ }^{3}$ Centre for Experimental Medicine, Wellcome-Wolfson Institute for Experimental Medicine, Queen's University of Belfast, Belfast, Northern Ireland

${ }^{4}$ Regional Intensive Care Unit, Royal Victoria Hospital, Belfast, Northern Ireland
}

Correspondence to Professor Daniel F McAuley, Centre for Experimental Medicine, Wellcome-Wolfson Institute for Experimental Medicine, Queen's University of Belfast, Belfast BT7 1NN, UK; d.f.mcauley@qub.ac.uk were selected to represent inflammation, coagulation and endothelial activation, as hallmarks of ARDS biology. ${ }^{6}$ The dataset was divided into a training cohort $(n=454$ patients) and validation cohort $(n=246$ patients), based on the study recruitment period. Cluster analysis was used to identify homogenous ARDS subphenotypes in the training cohort. ${ }^{10}$ The most predictive biomarkers were then confirmed in the validation cohort. These biological clusters were then linked to clinical and outcome characteristics of ARDS patients to derive clinical subphenotypes, namely reactive and uninflamed. These two clinical ARDS subphenotypes differed in terms of illness severity and critical care mortality, with the reactive group having a greater risk of death.

A key question for the reader is whether these associations are spurious or indirect or causal? ${ }^{11}$ Cluster analysis methods generate different results dependent on the variables chosen for identifying similarities between patients and the method of clustering. ${ }^{10}$ Bos et al chose biomarker characteristics as the variables on which the groups should be similar and used Ward's method of agglomerative hierarchical clustering to identify two potentially generalisable ARDS clusters. Hierarchical clustering is a commonly used iterative method to identify homogenous groups or clusters based on specific characteristics. The basic algorithm starts with assigning each ARDS patient a 'value' based on their individual biomarker profile. Then patients with similar 'values' are grouped together to form clusters. The underlying principle is that ARDS patients within each cluster will have similar biomarker profiles and that between clusters biomarker profiles will be different. Depending on the parameters specified, the same dataset can result in potentially different results with different clustering algorithms and there are no universally agreed optimal rule(s) for clustering. ${ }^{10}$ Another potential limitation is that only patients with data on all chosen biomarkers were included and missing data in clinical variables were imputed, which has the potential for selection and information bias. The blood sampling window for biomarker measurement in this cohort was wide and drawn either on the day of ARDS diagnosis or the day before or the day after, challenging the time-based arguments for causal relationships. Despite these challenges, Bos et al provide important data with strong associations, that are consistent with our current knowledge, have biological plausibility and external validity.

Calfee and colleagues have led the field in defining ARDS subphenotypes. Using latent class analysis of clinical and biomarker data from patients enrolled in ARDS randomised controlled trials, Calfee et al have originally identified two ARDS subphenotypes. ${ }^{12} 13$ The reactive subphenotype identified by Bos et al shares many of the features of the hyperinflammatory ARDS subphenotype reported previously, ${ }^{12}{ }^{13}$ although the proportion of patients in the reactive group is much higher than the hyperinflammatory subphenotype. This suggests that the hyperinflammatory and reactive groups may represent a similar subphenotype, although this is unproven. The findings from Bos et al are significant in that they have identified comparable subphenotypes in an observational cohort of patients with ARDS using a different analytic approach. While Calfee et al identified these ARDS subphenotypes using clinical and biomarker data, Bos et al identified them purely on biomarker data. The blood sampling window for biomarker measurement in these studies was defined from trial enrolment (which could be up to 2 days after meeting ARDS criteria in the ARDSnet trials), which also challenges the time-based arguments for causal relationships. Furthermore, it is possible that in the study by Bos et al, ${ }^{9}$ ARDS subjects were sampled earlier than in the ARDSnet trials, which may be a potential explanation for the higher proportion of 'reactive' subphenotype in this study. It would be important to test whether similar subphenotypes emerge after harmonising these different study datasets and performing both cluster and latent class analyses. Table 1 provides a comparative summary of these three studies.

Several important questions remain unanswered. First, assuming the hyperinflammatory/reactive subphenotype represents a common subphenotype, further work is needed to identify the key discriminant makers that reliably define this ARDS subset. Ideally, a minimal dataset of variables could be identified to efficiently achieve this. Second, although it remains unknown if ARDS subphenotypes 
Table 1 Summary of studies that report ARDS subphenotypes

\begin{tabular}{|c|c|c|c|}
\hline Parameter & Bos et $a l^{9}$ & Calfee C et $a l^{13}$ & Famous et $a l^{12}$ \\
\hline Sample size & 700 & 1022 & 1000 \\
\hline Recruitment period & 2011-2013 & 1996-2002 & 2000-2005 \\
\hline Study design & Observational cohort & $\mathrm{RCT}$ analysed as cohort & $\mathrm{RCT}$ analysed as cohort \\
\hline ARDS P/F criteria & $\leq 300$ & $<300$ & $<300$ \\
\hline Blood sampling & Around ARDS diagnosis & At baseline & At baseline \\
\hline $\begin{array}{l}\text { Biomarkers used for } \\
\text { deriving sub-phenotypes }\end{array}$ & $\begin{array}{l}\text { Lung epithelial: none } \\
\text { Endothelial: E-selectin; P-selectin; ANG1/2 } \\
\text { Coagulation: antithrombin; D-Dimer; tPA; PAI-1; } \\
\text { Inflammation: fractalkine; GM-CSF; ICAM-1; IFN- } \gamma \text { I IL-1 } \beta \text {; IL-6; } \\
\text { IL-8; IL-10; IL-13; TNF- } \alpha \text {; MMP-8; TIMP-1; }\end{array}$ & $\begin{array}{l}\text { Lung epithelial: SP-D } \\
\text { Endothelial: ICAM-1; vWF } \\
\text { Coagulation: protein C; PAI-1 } \\
\text { Inflammation: sTNFR-1; IL-6; IL-8 }\end{array}$ & $\begin{array}{l}\text { Lung epithelial: SP-D } \\
\text { Endothelial: ICAM-1; vWF; ANG-2 and } \\
\text { RAGE } \\
\text { Coagulation: protein C; PAI-1 } \\
\text { Inflammation: sTNFR-1; IL-6; IL-8 }\end{array}$ \\
\hline $\begin{array}{l}\text { Clinical variables } \\
\text { used for deriving } \\
\text { subphenotypes }\end{array}$ & None & $\begin{array}{l}\text { Age, gender, ethnicity, BMI, respiratory*; } \\
\text { cardiovasculart; creatinine; urine output; } \\
\text { bilirubin; temperature; haematocrit; } \\
\text { WBC count; sodium; glucose; albumin; } \\
\text { platelets; bicarbonate; aetiology of ARDS‡ }\end{array}$ & $\begin{array}{l}\text { Age, gender, ethnicity, BMI, respiratory"; } \\
\text { cardiovasculart; creatinine; urine output; } \\
\text { bilirubin; temperature; haematocrit; WBC } \\
\text { count; sodium; glucose; albumin; platelets; } \\
\text { bicarbonate; aetiology of ARDS } \ddagger\end{array}$ \\
\hline $\begin{array}{l}\text { Analytical approach to } \\
\text { derive ARDS subsets }\end{array}$ & Cluster analyses based only on biomarker data & $\begin{array}{l}\text { Latent class analyses based grouping } \\
\text { based on clinical and biomarker data }\end{array}$ & $\begin{array}{l}\text { Latent class analyses based grouping based } \\
\text { on clinical and biomarker data }\end{array}$ \\
\hline $\begin{array}{l}\text { ARDS subset (prevalence } \\
\%)\end{array}$ & $\begin{array}{l}\text { Reactive phenotype }(58.0 \%) \\
\text { versus } \\
\text { Uninflamed }(42.0 \%)\end{array}$ & $\begin{array}{l}\text { Hyperinflammatory }(29.4 \%) \\
\text { versus } \\
\text { Phenotype } 1(70.6 \%)\end{array}$ & $\begin{array}{l}\text { Hyperinflammatory }(27.3 \%) \\
\text { versus } \\
\text { Phenotype } 1(72.7 \%)\end{array}$ \\
\hline $\begin{array}{l}\text { Mortality (\%) by ARDS } \\
\text { subset }\end{array}$ & $\begin{array}{l}\text { Reactive phenotype }=36.8 \% \\
\text { versus } \\
\text { Uninflamed }=14.9 \%\end{array}$ & $\begin{array}{l}\text { Hyperinflammatory=47.3\% } \\
\text { versus } \\
\text { Phenotype } 1=19.4 \%\end{array}$ & $\begin{array}{l}\text { Hyperinflammatory=45.0\% } \\
\text { versus } \\
\text { Phenotype } 1=22.0 \%\end{array}$ \\
\hline $\begin{array}{l}\text { Discriminant markers } \\
\text { between phenotypes }\end{array}$ & IL-6; IFN- $\gamma$, ANG1/2; PAI-1 & IL-6; sTNFR1; vasopressor use; IL-8; HCO3 & $\begin{array}{l}\text { IL-8; sTNFR1; vasopressor use; } \mathrm{HCO} \text {; } \\
\text { minute ventilation }\end{array}$ \\
\hline
\end{tabular}

The table shows the summary of three recent studies that report ARDS subphenotypes. The Respiratory system variables* included minute ventilation, mean airway pressure, plateau pressure, respiratory rate, tidal volume, positive end-expiratory pressure; partial pressure $\mathrm{PaO} 2$ of carbon dioxide ( $\mathrm{PaCO} 2$ ) and $\mathrm{PaO2} / \mathrm{FiO2}$ ratio.

The Cardiovasculart system variables include highest heart rate, lowest systolic blood pressure and vasopressor use.

The aetiology of ARDS $\ddagger$ was coded as trauma, sepsis, aspiration, pneumonia or other.

ANG1/2, angiopoietin 1 and 2; ARDS, acute respiratory distress syndrome; BMI, body mass index; GM-CSF, granulocyte-monocyte colony stimulating factor; $\mathrm{HCO}_{3}$, bicarbonate; ICAM-1, intracellular adhesion molecule-1; IFN- $\gamma$, interferon gamma; IL, interleukins 6, 8, 10, 13; IL-1 $\beta$, interleukin-1 beta; MMP-8, matrix metalloproteinase-8; P/F, Pa02/FiO2 ratio; PAI-1, plasminogen activator inhibitor-1; RAGE, receptor for advanced glycation end products; RCT, randomised controlled trial; SP-D, surfactant protein-D; sTNFR-1, soluble tumour necrosis factor receptor-1; TIMP-1, tissue inhibitor of metalloproteinase-1; TNF- $\alpha$, tumour necrosis factor-alpha; tPA, tissue plasminogen activator; vWF, von-Williebrand's factor; WBC, white blood cell count.

respond differently to pharmacotherapies, for developing pharmacotherapies targeted at the hyperinflammatory/reactive subphenotype we need to determine the stability of the ARDS subgroup over time. This is important to identify the therapeutic window for interventions targeted at this subphenotype. In addition, it would be important to define if and how moving from this subphenotype to an uninflamed phenotype represents therapeutic success or failure to guide ongoing treatment. Third, development of pointof-care assays along with algorithms to define these ARDS subphenoytpes at the bedside in real time is essential to enable this information to inform clinical trials targeting these subphenotypes.

In summary, ARDS continues to be a clinical and research challenge in terms of developing pharmacological therapies. Bos et al provide intriguing data that highlights the need for further work to identify ARDS subsets with defined treatable traits. These subphenotypes should be based on modifiable biological characteristics linked to both the risk of poor outcomes and response to the tested treatment. This will enable personalised care of patients with ARDS.

Contributors MS-H wrote the first draft. MS-H and DFM critically revised the manuscript for important intellectual content and agreed on the final submitted version of the manuscript.

Funding MS-H is supported by the National Institute for Health Research Clinician Scientist Award (NIHRCS-2016-16-011).

Disclaimer The views expressed in this publication are those of the author(s) and not necessarily those of the NHS, the UK National Institute for Health Research or the Department of Health.

\section{Competing interests None declared.}

Provenance and peer review Commissioned; externally peer reviewed.

(C) Article author(s) (or their employer(s) unless otherwise stated in the text of the article) 2017. All rights reserved. No commercial use is permitted unless otherwise expressly granted.

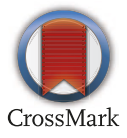

To cite Shankar-Hari M, McAuley DF. Thorax 2017;72:867-869.

Published Online First 17 July 2017

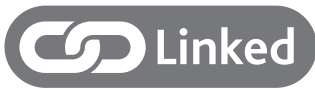

http://dx.doi.org/10.1136/thoraxjnl-2016-209719

Thorax 2017:72:867-869.

doi:10.1136/thoraxjnl-2017-210422

\section{REFERENCES}

1 Ranieri VM, Rubenfeld GD, Thompson BT, et al. Acute respiratory distress syndrome: the Berlin definition. JAMA 2012;307:2526-33.

2 Bellani G, Laffey JG, Pham T, et al. Epidemiology, patterns of care, and mortality for patients with acute respiratory distress syndrome in intensive care units in 50 countries. JAMA 2016;315:788-800.

3 Phua J, Badia JR, Adhikari NK, et al. Has mortality from acute respiratory distress syndrome decreased over time?: A systematic review. Am J Respir Crit Care Med 2009:179:220-7.

4 Boyle AJ, Mac Sweeney R, McAuley DF. Pharmacological treatments in ARDS; a state-of-the-art update. BMC Med 2013;11:166.

5 Pham T, Rubenfeld GD. Fifty Years of Research in ARDS. The Epidemiology of Acute Respiratory Distress 
Syndrome. A 50th Birthday Review. Am J Respir Crit Care Med 2017;195:860-70.

6 Sweeney RM, McAuley DF. Acute respiratory distress syndrome. Lancet 2016;388:2416-30.

7 Shankar-Hari M, Rubenfeld GD. The use of enrichment to reduce statistically indeterminate or negative trials in critical care. Anaesthesia 2017;72:560-5.

8 Prescott HC, Calfee CS, Thompson BT, et al. Toward smarter lumping and smarter splitting: rethinking strategies for sepsis and acute respiratory distress syndrome clinical trial design. Am J Respir Crit Care Med 2016:194:147-55.

9 Bos LD, Schouten LR, van Vught LA, et al. Identification and validation of distinct biological phenotypes in patients with acute respiratory distress syndrome by cluster analysis. Thorax 2017;72:876-83.

10 Frades I, Matthiesen R. Overview on techniques in cluster analysis. Methods Mol Biol 2010;593:81-107.

11 Grimes DA, Schulz KF. Bias and causal associations in observational research. Lancet 2002;359:248-52.
12 Famous KR, Delucchi K, Ware LB, et al. Acute respiratory distress syndrome subphenotypes respond differently to randomized fluid management strategy. Am J Respir Crit Care Med 2017;195:331-8

13 Calfee CS, Delucchi K, Parsons PE, et al. Subphenotypes in acute respiratory distress syndrome: latent class analysis of data from two randomised controlled trials. Lancet Respir Med 2014;2:611-20. 